\title{
Considérations sur le vortex de Hill
}

\section{Hill's vortex considérations}

\section{Roger Prud'homme ${ }^{1}$}

${ }^{1}$ Directeur de Recherche Emérite, UMR 7190 - Sorbonne-Université / Centre National de la Recherche Scientifique

RÉSUMÉ. On examine des cas de tourbillons stationnaires pouvant apparaître à l'intérieur de gouttes liquides sphériques. Le premier cas est celui d'un écoulement extérieur incompressible de vitesse uniforme à l'infini, entrainant par frottement le liquide de la goutte vers la constitution d'un vortex de Hill. Dans le second cas, le fluide extérieur n'interagit pas avec le liquide, mais la goutte est soumise à un gradient de température axial provoquant une variation de tension superficielle. Cette fois c'est le mouvement induit qui entraine le liquide intérieur. On remarque que les deux situations peuvent conduire au même vortex de Hill. Des effets combinés sont envisagés. On s'intéresse par ailleurs au facteur temps dans ces phénomènes.

ABSTRACT. We examine cases of stationary vortices that can appear inside spherical liquid drops. The first case is that of an incompressible external flow of uniform speed at infinity, leading the liquid in the drop by friction to form a Hill vortex. In the second case, the external fluid does not interact by friction with the liquid, but the drop is subjected to an axial temperature gradient causing a variation in surface tension. This time it is the induced movement which entrains the internal liquid. Note that the two situations can lead to the same Hill vortex. Combined effects are envisioned. We are also interested in the time factor in these phenomena.

MOTS-CLÉS. gouttes liquides, instabilités, champ de vitesse, champ thermique, vortex de Hill.

KEYWORDS. liquid drops, instabilities, velocity field, thermal field, Hill vortex.

\section{Liste des symboles}

\begin{tabular}{|c|c|c|c|}
\hline Symbole & Signification & Symbole & Signification \\
\hline$D$ & Débit d'une source & $\begin{array}{c}(x, y, z) \\
\left(x_{1}, x_{2}, x_{3}\right)\end{array}$ & Coordonnées cartésiennes \\
\hline $\begin{array}{c}\left(\overrightarrow{\mathbf{e}}_{r}, \overrightarrow{\mathbf{e}}_{\theta}, \overrightarrow{\mathbf{e}}_{\varphi}\right) \\
(\overrightarrow{\mathbf{i}}, \overrightarrow{\mathbf{j}}, \overrightarrow{\mathbf{k}})\end{array}$ & Vecteurs de base unitaires & $Y_{l}^{m}(\theta, \varphi)$ & Harmonique sphérique \\
\hline$K$ & Intensité d'un doublet & $\alpha, \alpha_{e}, \alpha_{i}$ & Coefficients de fonction de courant \\
\hline $\overrightarrow{\mathbf{n}}$ & Normale unitaire & $\phi$ & Potentiel de vitesse \\
\hline$p, p_{\infty}$ & Pression, $p$ à l'infini & $\varepsilon$ & Taux de déformation \\
\hline$P_{l, m}$ & Polynôme de Legendre & $\Gamma$ & Intensité d'un tourbillon irrotationnel \\
\hline$r, \theta, \varphi$ & Coordonnées sphériques & $\mu, v=v / \rho$ & Coefficients de viscosité de cisaillement \\
\hline$R$ & Constante des gaz, rayon d'une sphère & $\rho$ & Masse volumique \\
\hline$s$ & Abscisse curviligne & $\sigma$ & Tension de surface \\
\hline$S$ & Surface & $\Omega$ & Vitesse de rotation \\
\hline$T$ & Température absolue & $\overrightarrow{\mathbf{\omega}}$ & Vecteur tourbillon \\
\hline $\overrightarrow{\mathbf{t}}$ & Tangente unitaire & $\psi$ & Fonction de courant \\
\hline$U, U_{\infty}$ & Vitesse en surface, vitesse à l'infini & i, e & Ecoulement resp. interne, externe \\
\hline $\overrightarrow{\mathbf{v}}, v=|\overrightarrow{\mathbf{v}}|$ & Vecteur vitesse, module de vitesse & $\mathrm{t}, \mathrm{n}$ & Resp. tangent, normal à la sphère de rayon $r$ \\
\hline$(u, v)$ ou $\left(v_{r}, v_{\theta}\right)$ & Composantes du vecteur vitesse & $r, \theta$ & Resp. radial, tangentiel \\
\hline
\end{tabular}




\section{Introduction}

Les gouttes des sprays subissent des actions diverses dépendant de leur origine et de la situation physique résultante dans laquelle elles se trouvent : spray d'arrosage, pulvérisation médicale, injecteur de moteur automobile, injecteur de moteur de fusée, etc.

Leur modélisation comprend d'une part l'examen à l'échelle de chaque goutte individuelle, et d'autre part l'étude du spray lui-même à plus grande échelle, en tant que constituant d'un écoulement multiphasique le plus souvent liquide-gaz.

La goutte individuelle est souvent considérée comme sphérique. C'est le cas des petites gouttes où les efforts capillaires sont décisifs pour l'établissement de la sphéricité. Cette simplicité de forme géométrique est remise en cause dans le cas de grosses gouttes et dès qu'elles sont soumises à des efforts importants d'origine aérodynamique par exemple. Par soucis de simplicité, on essaye lors des recherches théoriques, de garder la forme sphérique le plus longtemps possible.

Les échanges entre la goutte individuelle et son environnement gazeux sont évidemment différents suivant qu'il y a évaporation-condensation ou non. Ils concernent les masses des constituants, la quantité de mouvement, et l'énergie. Chaque phase en présence subit également des mouvements et des phénomènes de transfert.

Pour l'écoulement du spray proprement dit, l'on est souvent amené à modéliser ce qui se passe à l'intérieur des gouttes individuelles. Le plus classique est de caractériser ces dernières par leur rayon, leur masse, leur température et leur vitesse. La distribution en diamètre et en vitesse des gouttes restera toujours l'élément capital.

Néanmoins, il peut être intéressant de s'occuper des mouvements internes aux gouttes de taille moyenne car ceux-ci agissent sur les échanges au niveau leur surface. C'est le problème qui est proposé dans cet article, où nous aurons donc à étudier simultanément les écoulements extérieur et intérieur de gouttes individuelles.

Nous avons retenu ici le cas de gouttes liquides sphériques soumises, soit à un écoulement gazeux extérieur uniforme, soit à un gradient thermique dans une direction axiale. Nous verrons que la modélisation en tourbillon de Hill est une solution intéressante pour les écoulements intérieurs.

L'un des problèmes majeurs est celui du raccordement entre l'écoulement intérieur à la sphère limitant la goutte et l'écoulement du fluide extérieur.

En effet, si l'on fait l'hypothèse d'un fluide parfait à l'extérieur, on peut être conduit logiquement à admettre des conditions de glissement parfait pour ce fluide au niveau de la surface de la goutte. Mais alors comment admettre qu'il y ait entrainement du liquide intérieur à la goutte par le fluide extérieur?

Le problème des efforts exercés sur la goutte par le fluide extérieur est d'ailleurs posé en ce qui concerne leur résultante que l'on trouve nulle! Cela constitue le célèbre paradoxe de Dalembert. L'on est alors invité à prendre en compte la viscosité du fluide extérieur, au moins dans le proche voisinage de la sphère, ce qui conduit à la théorie de Stokes dans le cas de la sphère rigide. Les résultats doivent d'ailleurs être modifiés pour tenir compte d'une sphère liquide. Et en présence d'évaporation-condensation du liquide c'est encore plus compliqué!

Enfin on sait que pour de nombreux problèmes linéaires on peut superposer des solutions élémentaires (voir l'annexe A1). Nous le ferons à chaque fois que c'est possible, en prenant en compte la nature des fluides et les domaines de validité que sont l'intérieur et l'extérieur de la goutte. 


\section{Goutte liquide sphérique soumise à un écoulement extérieur uniforme à l'infini}

Le tourbillon de Hill est utilisé pour modéliser l'écoulement à l'intérieur d'une goutte liquide sphérique en présence d'un écoulement relatif ${ }^{i}$.

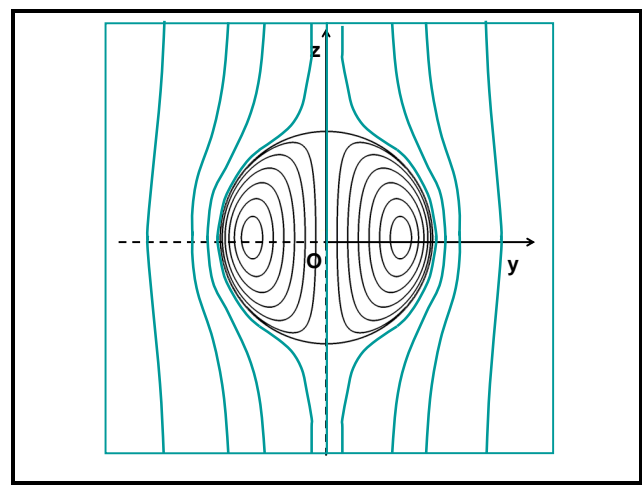

Figure 1. Goutte liquide en présence d'un écoulement uniforme à l'infini : allure des lignes de courant des écoulements externe et interne dans un plan passant par l'axe de symétrie.

Ce tourbillon est un cas particulier de mouvement stationnaire de révolution d'un fluide parfait incompressible ii. C'est un mouvement rotationnel à l'intérieur d'une sphère se comportant avec un écoulement extérieur irrotationnel, de telle sorte qu'en choisissant convenablement la constante multiplicative $\alpha$ de la fonction de courant, les vitesses des deux écoulements sont identiques à la surface de la sphère ${ }^{\text {iii }}$.

Nous rappelons d'abord les équations de l'écoulement fluide extérieur à la sphère de rayon $R$ et nous déterminerons ensuite le champ de vitesse de l'écoulement liquide stationnaire compatible ${ }^{1}$ intérieur à cette même sphère ${ }^{\mathrm{iv}}$.

L'écoulement stationnaire d'un fluide parfait à l'intérieur d'une sphère de rayon $R$ et l'écoulement autour de cette sphère sont des exemples caractéristiques, représentés sur la figure 1. Une présentation classique de l'écoulement extérieur est faite en annexe.

\subsection{Fluides incompressibles en coordonnées sphériques.}

\subsubsection{Présentation du système de coordonnées}

Les quantités r, $\theta, \varphi$ sont les coordonnées sphériques, représentées sur la figure 2.

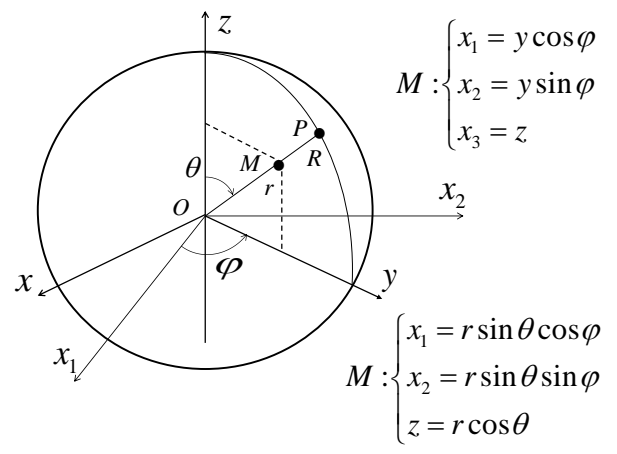

Figure 2. Définition des coordonnées sphériques et représentation d'une sphère de rayon $R$. Le point courant M peut être à l'intérieur ou à l'extérieur de la sphère considérée. $P$ est le point de la demi-droite OM situé à la surface de la sphère.

\footnotetext{
1 C'est-à-dire sans discontinuité de vitesse à l'interface.
} 
Dans le cas d'une symétrie autour de l'axe Oz, on travaille dans le plan à $\varphi=C t e$ de la figure 2 puisque le mouvement est indépendant de cet angle. On peut y définir les vecteurs unitaires de base.

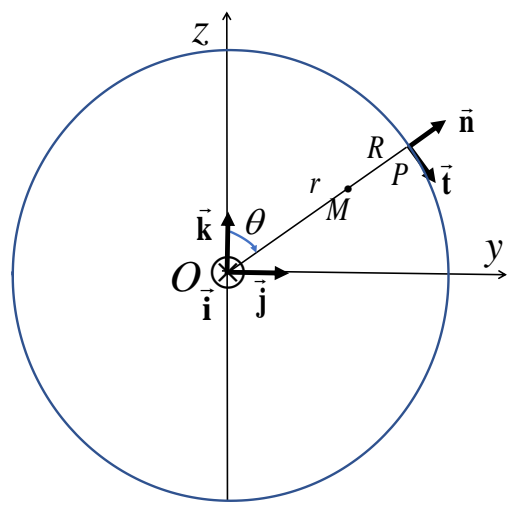

$$
\begin{gathered}
M\left\{\begin{array}{l}
y=r \sin \theta \\
z=r \cos \theta
\end{array}\right. \\
P\left\{\begin{array}{l}
y=R \sin \theta \\
z=R \cos \theta
\end{array}\right. \\
\left\{\begin{array}{l}
\overrightarrow{\mathbf{i}}=\overrightarrow{\mathbf{j}} \times \overline{\mathbf{k}} \\
\overrightarrow{\mathbf{j}}=\overrightarrow{\mathbf{t}} \cos \theta+\overrightarrow{\mathbf{n}} \sin \theta \\
\overrightarrow{\mathbf{k}}=-\overrightarrow{\mathbf{t}} \sin \theta+\overrightarrow{\mathbf{n}} \cos \theta
\end{array}\right.
\end{gathered}
$$

Figure 3. Définition des coordonnées dans le plan y0z contenant l'axe de symétrie Oz. Au point $P$ de la surface de la sphère de rayon $R$, on définit les vecteurs unitaires tangent $\overrightarrow{\mathbf{t}}$ et normal $\overrightarrow{\mathbf{n}}$ au grand cercle considéré. Ces vecteurs correspondent aux vecteurs de base $\left(\overrightarrow{\mathbf{e}}_{\theta}, \overrightarrow{\mathbf{e}}_{r}\right)$ au point considéré.

\subsubsection{Equation de continuité}

L'équation de continuité d'un fluide incompressible s'écrit en notations vectorielles $: \vec{\nabla} \cdot \overline{\mathbf{v}}=0$. Sous forme développée, cela donne :

$$
\frac{1}{r^{2}} \frac{\partial}{\partial r}\left(r^{2} v_{r}\right)+\frac{1}{r \sin \theta} \frac{\partial}{\partial \theta}\left(\sin \theta v_{\theta}\right)+\frac{1}{r \sin \theta} \frac{\partial v_{\varphi}}{\partial \varphi}=0
$$

Dans le cas d'une symétrie d'axe $O z$, le mouvement est identique dans chaque plan contenant cet axe.

Les valeurs des diverses grandeurs du fluide ne dépendent plus de l'angle $\varphi$. L'équation de continuité se réduit donc à : $\frac{1}{r^{2}} \frac{\partial}{\partial r}\left(r^{2} v_{r}\right)+\frac{1}{r \sin \theta} \frac{\partial}{\partial \theta}\left(\sin \theta v_{\theta}\right)=0$, et l'on peut alors définir le champ des vitesses à partir de la fonction de courant $\psi$ comme suit :

$$
v_{r}=-\frac{1}{r^{2} \sin \theta} \frac{\partial \psi}{\partial \theta}, \quad v_{\theta}=\frac{1}{r \sin \theta} \frac{\partial \psi}{\partial r}, \quad v_{\varphi}=0
$$

\subsubsection{Expressions de la fonction de courant en présence d'une sphère}

On considère deux sortes d'écoulements : un écoulement est irrotationnel (e) à l'extérieur de la sphère de rayon $R$, et un écoulement rotationnel (i) de vorticité $\omega$ à l'intérieur de la sphère. Les écoulements se raccordent en tout point de leur frontière sphérique.

En ce qui concerne la vorticité, nulle à l'extérieur, on démontre que $\omega=5 \alpha_{i} y$ en vertu de la loi de transport du vecteur tourbillon $\overrightarrow{\boldsymbol{\omega}}$ du fluide intérieur.

Dans le système de coordonnées sphériques décrites ci-dessus, on a : $r^{2}=y^{2}+z^{2}$. 
Les fonctions de courant $\psi_{e}$ et $\psi_{i}$ autour et à l'intérieur de la sphère de rayon $R$ s'expriment comme suit 2:

$$
\left\{\begin{array}{l}
\psi_{e}=\alpha_{e} y^{2}\left(1-\frac{R^{3}}{r^{3}}\right), \text { avec } \alpha_{e}=V_{0} / 2 \text { pour } r \geq R, \text { et } \\
\psi_{i}=\alpha_{i} y^{2}\left(R^{2}-r^{2}\right), \text { avec } \alpha_{i}=-3 V_{0} / 4 R^{2} \text { pour } r \leq R
\end{array}\right.
$$

donc :

$$
\left\{\begin{array}{l}
\psi_{e}=\frac{V_{0}}{2} y^{2}\left(1-\frac{R^{3}}{r^{3}}\right) \quad \text { pour } \mathrm{r} \geq R \\
\psi_{i}=-\frac{3 V_{0}}{4} y^{2}\left(1-\frac{r^{2}}{R^{2}}\right) \quad \text { pour } r \leq R
\end{array} .\right.
$$

Dans le problème du mouvement autour d'une sphère immobile de rayon $R$, la vitesse $V_{0}$ est la vitesse à l'infini de l'écoulement extérieur : $V_{0}=U_{\infty}$.

Les calculs effectués avec la fonction de courant sont résumés dans le tableau 1. Ils sont explicités ci-après.

\begin{tabular}{|c|c|c|}
\hline & $\begin{array}{c}\text { Ecoulement intérieur (vortex } \\
\text { de Hill) } r \leq R\end{array}$ & Ecoulement extérieur $r \geq a$ \\
\hline Fonction de courant & $\psi_{i}=\alpha_{i} r^{2} \sin ^{2} \theta\left(R^{2}-r^{2}\right)$ & $\psi_{e}=\alpha_{e} r^{2} \sin ^{2} \theta\left(1-R^{3} / r^{3}\right)$ \\
\hline Vitesse $\overrightarrow{\mathbf{v}}$ & $\left\{\begin{array}{l}v_{r i}=-2 \alpha_{i}\left(R^{2}-r^{2}\right) \cos \theta \\
v_{\theta i}=2 \alpha_{i}\left(R^{2}-2 r^{2}\right) \sin \theta\end{array}\right.$ & $\left\{\begin{array}{l}v_{r e}=-2 \alpha_{e}\left(1-R^{3} / r^{3}\right) \cos \theta \\
v_{\theta e}=2 \alpha_{e}\left(1+R^{3} / 2 r^{3}\right) \sin \theta\end{array}\right.$ \\
\hline Coefficients & $\alpha_{i}=U_{S} / 2 R^{2}=-3 V_{0} / 4 R^{2}$ & $\alpha_{e}=-U_{S} / 3=V_{0} / 2$ \\
\hline
\end{tabular}

Tableau1. Correspondance entre les coefficients pour un écoulement extérieur venant des $x$ négatifs avec le module de vitesse $U_{\infty}$. $U_{S}$ est la vitesse à la surface de la sphère en $z=0$. Ce tableau intègre les résultats de la section 2 .

\subsubsection{Ecoulement extérieur à la sphère}

L'écoulement stationnaire de fluide parfait à l'extérieur de la sphère est un écoulement irrotationnel. Sa fonction de courant (Figure 3) est (indice $e$ pour extérieur) :

$$
\psi_{e}=\alpha_{e} r^{2} \sin ^{2} \theta\left(1-\frac{R^{3}}{r^{3}}\right), \quad r \geq R, \quad \alpha_{e}=\frac{U_{\infty}}{2}
$$

Les composantes du vecteur vitesse sont :

$$
v_{r e}=-2 \alpha_{e}\left(1-R^{3} / r^{3}\right) \cos \theta, \quad v_{\theta e}=2 \alpha_{e}\left(1+R^{3} / 2 r^{3}\right) \sin \theta
$$

2 Réf. iii, p. 312. 
Ses lignes de courant sont représentées sur la figure 3 avec :

$$
\bar{z}=z / R, \bar{y}=y / R, \bar{\psi}_{e}=2 \psi_{e} / U_{\infty} R^{2} .
$$

Vitesse maximale :

Appelons $U_{S}$ la valeur de la vitesse en $z=0, y=R$, ce qui correspond à $r=R, \theta=\pi / 2$. On trouve : $U_{S i}=U_{z i}=2 \alpha_{i} R^{2}, U_{y i}=0$. Si on veut que $U_{S}$ soit positif, il faut donc que $\alpha$ soit positif. On a alors : $\alpha_{i}=U_{S} / 2 R^{2}$ et la fonction de courant s'écrit : $\psi_{i}=\frac{U_{S}}{2 R^{2}} r^{2} \sin ^{2} \theta\left(R^{2}-r^{2}\right) . U_{S}$ est la valeur maximum de $U$ à la surface de la sphère.

Les vecteurs vitesse de cet écoulement et de l'écoulement intérieur seront identiques en tout point de la surface de la sphère si l'on choisit convenablement la vitesse à l'infini $U_{r e}(\infty)=-U_{r e}(-\infty)=U_{\infty}$.

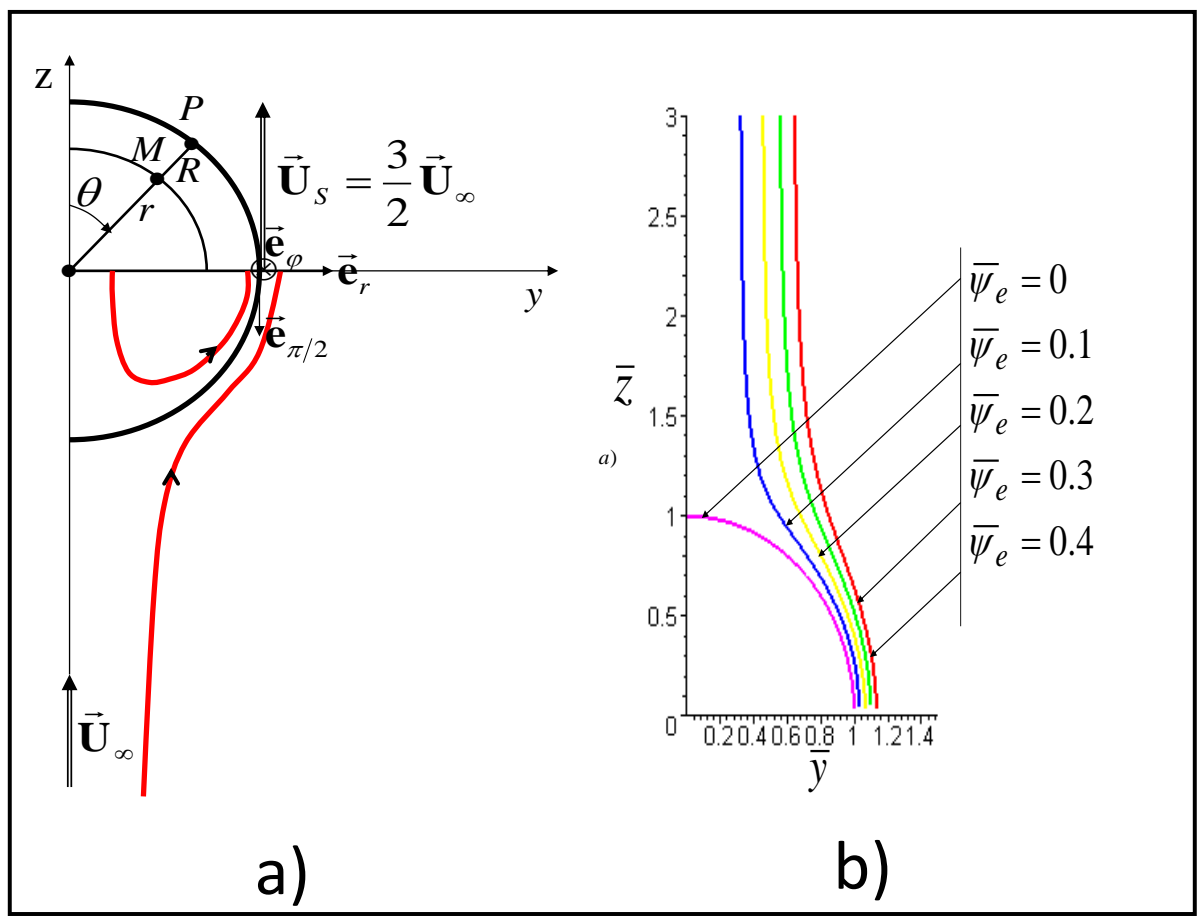

Figure 3. a) Lignes de courant intérieure et extérieure, vecteurs vitesse à l'infini $\vec{U}_{\infty}$ et maximale $\vec{U}_{s}$ à la surface. b) Lignes de courant de l'écoulement extérieur à la sphère dans le quart de plan supérieur passant par l'axe. $\bar{z}=\bar{z}(\bar{y})$, pour différentes valeurs de la fonction de courant réduite $\bar{\psi}_{e}=2 \psi_{e} / U_{\infty} R^{2}$.

\subsection{Ecoulement intérieur à la sphère}

\subsubsection{Vortex de Hill}

La fonction de courant de la forme :

$$
r \leq R, \quad \psi_{i}=\alpha_{i} r^{2} \sin ^{2} \theta\left(R^{2}-r^{2}\right)
$$

correspond à un écoulement intérieur rotationnel (réf. iii).

Des éléments sur les écoulements rotationnels de fluides parfaits rotationnels sont donnés dans l'annexe A2.

Connaissant la fonction de courant de l'écoulement intérieur, on trouve les composantes suivantes radiale et angulaire du vecteur vitesse : 
$v_{r i}=-2 \alpha_{i}\left(R^{2}-r^{2}\right) \cos \theta, \quad v_{\theta i}=2 \alpha_{i}\left(R^{2}-r^{2}\right) \sin \theta$

Les résultats sont représentés sur la figure 4.

Chaque valeur de la fonction de courant correspond à une surface torique. La valeur limite $\bar{\psi}_{i}=0$ est la sphère elle-même.

Les vecteurs vitesse de l'écoulement extérieur sont identiques à ceux du vortex de Hill en tout point de la surface de la sphère si l'on choisit convenablement les constantes $\alpha_{i}$ et $\alpha_{e}$ en rapport avec la vitesse à l'infini $U_{\infty}$ [on a : $v_{r e}(\infty)=-v_{r e}(-\infty)=U_{\infty}$ ].

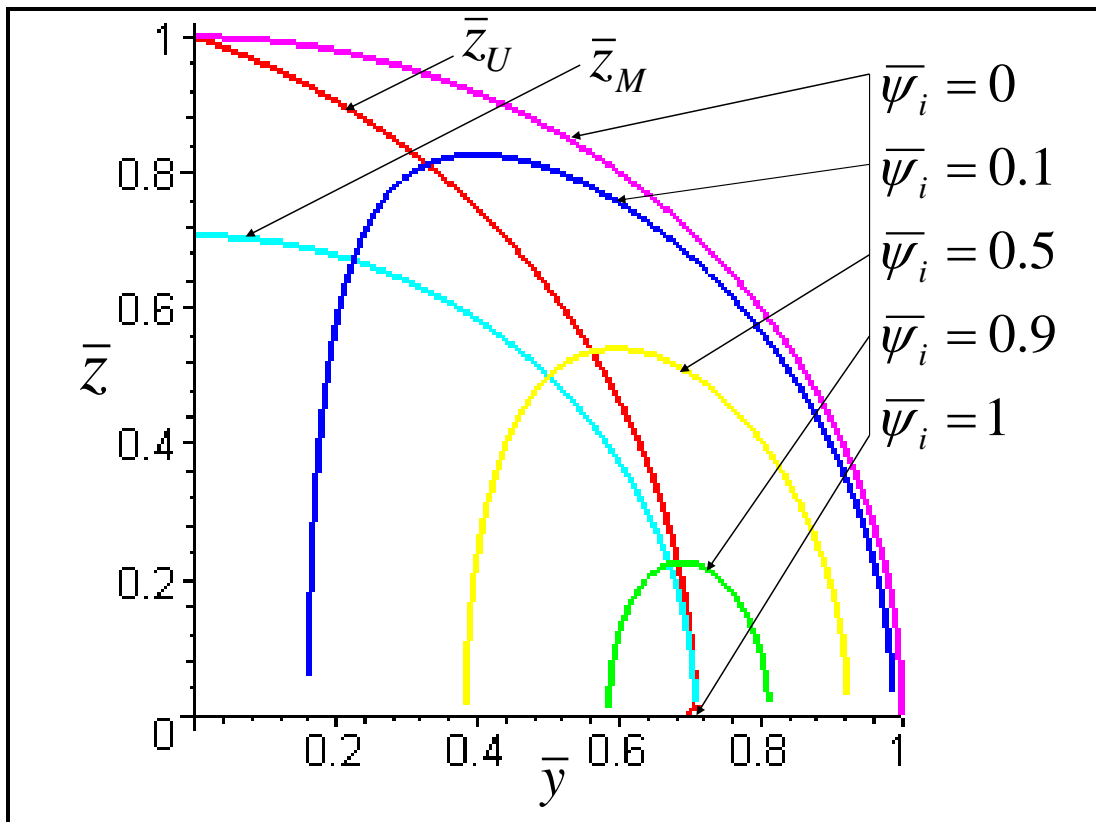

Figure 4. Lignes de courant calculées dans le quart de plan supérieur passant par l'axe. $\bar{z}=\bar{z}(\bar{y})$, $\bar{y}=y / R, \bar{z}=z / R$ pour plusieurs valeurs de $\bar{\psi}_{i}=4 \psi_{i} / \alpha_{i} R^{4} \cdot \bar{z}_{M}(\bar{y})$ correspond au lieu géométrique des points de contact des droites issues de $O$ tangentes aux lignes de courant. C'est un quart de cercle de rayon $R \sqrt{2} / 2 \cdot \bar{z}_{U}(\bar{y})$ est le lieu géométrique des points où la vitesse est minimum.

On trouve dans ce cas :

$$
\alpha_{i}=3 U_{\infty} / 4 R^{2}, \alpha_{e}=-U_{\infty} / 2
$$

Appelons $U_{S}$ la valeur de la vitesse maximale à la surface de la sphère, qui se produit en $y=R, z=0$, ce qui correspond à : $\theta=\pi / 2$. On trouve : $U_{S i}=2 \alpha_{i} R^{2}$. Si l'on veut que $U_{S}$ soit positif, il faut donc que $\alpha_{i}$ soit positif. On a alors : $\alpha_{i}=U_{S} / 2 R^{2}$ et la fonction de courant s'écrit :

$$
\psi_{i}=\frac{U_{S}}{2} r^{2} \sin ^{2} \theta\left(R^{2}-r^{2}\right)
$$

On sait donc déterminer le champ des vitesses et les surfaces de courant des écoulements internes et externes à la sphère (réf. iii). On a montré qu'alors les écoulements sont compatibles si $\alpha_{i}=3 U_{\infty} / 4 R^{2}$. La vitesse maximum à la surface de la sphère a pu alors être déterminée.

Le détail des calculs dans la goutte sphérique sont donnés en annexe à la section A3.

\subsubsection{Tourbillon en fluide visqueux}

L'équation de bilan du vecteur tourbillon en fluide visqueux incompressible s'écrit : 


$$
\partial \overrightarrow{\boldsymbol{\omega}} / \partial t+\vec{\nabla} \times(\vec{\nabla} \times \overrightarrow{\boldsymbol{\omega}})=v \Delta \overrightarrow{\boldsymbol{\omega}}
$$

Avec, en coordonnées sphériques :

$$
\omega=\frac{1}{2 r}\left[\frac{\partial\left(r v_{\theta}\right)}{\partial r}-\frac{\partial v_{r}}{\partial \theta}\right] \overrightarrow{\mathbf{e}}_{\varphi}
$$

\section{Ecoulement intérieur}

On trouve sans difficulté, pour le vortex de Hill : $\overrightarrow{\boldsymbol{\omega}}_{i}=-5 \alpha_{i} r \sin \theta \overrightarrow{\mathbf{e}}_{\varphi}$.

L'intensité $\omega_{i} \mathrm{du}$ vecteur tourbillon est proportionnelle à la distance $y=r \sin \theta$ à l'axe de symétrie. Le second terme du bilan du vecteur tourbillon se calcule comme suit. On pose : $\overrightarrow{\mathbf{A}}=\vec{\nabla} \times \overrightarrow{\boldsymbol{\omega}}$ et on obtient : $\quad A_{r}=10 \alpha_{i} r \sin ^{2} \theta\left(R^{2}-2 r^{2}\right), \quad A_{\theta}=10 \alpha_{i} r \sin \theta \cos \theta\left(R^{2}-r^{2}\right) . \quad$ On trouve alors : $\vec{\nabla} \times \overrightarrow{\mathbf{A}}=\vec{\nabla} \times(\vec{\nabla} \times \overrightarrow{\boldsymbol{\omega}})=\frac{1}{2 r}\left[\frac{\partial\left(r A_{\theta}\right)}{\partial r}-\frac{\partial A_{r}}{\partial \theta}\right] \overrightarrow{\mathbf{e}}_{\varphi}=\overrightarrow{\mathbf{0}}$.

En écoulement stationnaire $: \frac{\partial \overrightarrow{\boldsymbol{\omega}}}{\partial t}=\overrightarrow{\mathbf{0}}$. Il s'ensuit que le terme du second membre $v \Delta \overrightarrow{\boldsymbol{\omega}}$ est nul également.

La conséquence est que le vortex de Hill, solution de fluide parfait est également solution des équations des fluides visqueux.

Ecoulement extérieur

On a évidemment $\overrightarrow{\boldsymbol{\omega}}=\overrightarrow{\mathbf{0}}$ puisque l'écoulement est irrotationnel.

\section{Ecoulements d'une goutte liquide sphérique soumise à un gradient thermique axial}

\subsection{Présentation du problème}

L'effet d'un champ de température axial imposé sur les mouvements internes d'une goutte sphérique liquide a été étudié par divers auteurs. Ces mouvements sont causés par les variations de tension superficielle induites et par l'effet Marangoni qui en résulte en fluide visqueux. On peut citer en particulier Bauer v,vi,vii. Pour l'analyse mathématique, on a recours généralement aux harmoniques sphériques (voir Annexe A4).

Dans l'article de Bauer (1982), une goutte liquide flottant librement est soumise à sa surface à un champ de température axial induisant une convection thermique de Marangoni due à la variation de la tension superficielle. La fonction de courant et la distribution de vitesse sont déterminées analytiquement pour les champs de température stationnaires et instationnaires, en résolvant l'équation vérifiée par la fonction de courant à l'aide des fonctions de Legendre associées du premier type. Le cas particulier d'un champ de température axial linéaire stable est évalué numériquement.

On considère le cas de symétrie axiale $\mathrm{Oz}$, la goutte liquide étant centrée en $\mathrm{O}$.

L'équation de continuité de l'écoulement du liquide supposé incompressible s'écrit :

$$
\frac{1}{r^{2}} \frac{\partial}{\partial r}\left(r^{2} u\right)+\frac{1}{r \sin \theta} \frac{\partial}{\partial \theta}(v \sin \theta)=0
$$


où $u$ est la vitesse radiale et $v$ la vitesse angulaire. On peut donc introduire la fonction de courant $\psi$ telle que :

$$
u=-\frac{1}{r^{2} \sin \theta} \frac{\partial \psi}{\partial \theta}, v=\frac{1}{r \sin \theta} \frac{\partial \psi}{\partial r}
$$

En éliminant la pression des équations instationnaires de la quantité de mouvement, et en définissant l'opérateur :

$$
\bar{\Delta}=\frac{\partial^{2}}{\partial t^{2}}+\frac{\sin \theta}{r^{2}} \frac{\partial}{\partial \theta}\left(\frac{1}{\sin \theta} \frac{\partial}{\partial \theta}\right)
$$

il vient :

$$
\bar{\Delta}\left[\bar{\Delta} \psi-\frac{1}{v} \frac{\partial \bar{\psi}}{\partial t}\right]=0
$$

H.F. Bauer traite d'abord le cas stationnaire en supposant constante la dérivée de la tension superficielle $\sigma$ par rapport à la température $T$.

Cette température se développe en série de polynômes de Legendre suivant :

$$
T_{0}+T_{1} f_{0}(\xi)=\sum_{n=0}^{\infty} \alpha_{n} P_{n}(\xi)
$$

Avec $\xi=\cos \theta$ et la relation d'orthogonalité :

$$
\int_{\xi=-1}^{+1} P_{m}(\xi) P_{n}(\xi) d \xi=\left\{\begin{array}{l}
0 \text { pour } m \neq n \\
\frac{2}{2 n+1} \text { pour } m=n
\end{array}\right.
$$

La distribution de température à l'intérieur de la goutte sphérique est donnée par :

$$
T(r, \theta)=\sum_{n=0}^{\infty} \alpha_{n}\left(\frac{r}{R}\right)^{n} P_{n}(\cos \theta)
$$

avec des coefficients $\alpha_{n}$ vérifiant :

$$
\alpha_{n}=\left[T_{0} \int_{-1}^{+1} P_{n}(\xi) d \xi+T_{1} \int_{-1}^{+1} f_{0}(\xi) P_{n}(\xi) d \xi\right] \frac{2 n+1}{2}
$$

Dans le cas d'une température à la surface de la forme $T=T_{0}+T_{1} R \cos \theta$, on a $\alpha_{0}=T_{0}, \quad \alpha_{1}=T_{1} R, \quad \alpha_{n}=0$ pour $n>1$

La distribution de température à l'intérieur de la goutte est alors de la forme $T(r, \theta)=T_{0}+T_{1} r \cos \theta$, avec :

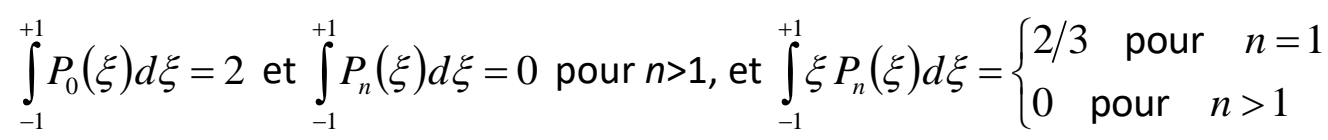


L'on résout donc l'équation ${ }^{\bar{\Delta}} \bar{\Delta} \psi=0$ avec la condition limite d'interface en $r=a$ :

$$
\tau_{r \theta}=\mu\left[r \frac{\partial}{\partial r}\left(\frac{v}{r}\right)+\frac{1}{r} \frac{\partial u}{\partial \theta}\right]=\frac{T_{1}}{a}\left|\frac{d \sigma}{d T}\right| f^{\prime}{ }_{0}(\xi) \sin \theta
$$

et les conditions d'annulation de flux :

$$
\int_{0}^{2 \pi} \int_{0}^{a} v \sin \theta_{0} r d \varphi d r=0 \text {, et } \int_{0}^{\pi} \int_{0}^{2 \pi} u_{r=r_{0}} r_{0}^{2} \sin \theta d \varphi d \theta=0
$$

Dans le cas de la température axiale linéaire $: T=T_{0}+T_{1} R \cos \theta$, on trouve la fonction de courant

$$
\psi(r, \theta)=-\frac{T_{1} R^{3}}{6 \mu}\left|\frac{d \sigma}{d T}\right|\left[\left(\frac{r}{R}\right)^{2}-\left(\frac{r}{R}\right)^{4}\right] \sin ^{2} \theta
$$

ce qui correspond au cas du tourbillon de Hill de la section 2.2.

Bauer résout aussi le problème dans le cas d'un champ axial de température quelconque stationnaire, ou avec une dépendance périodique en temps.

\subsection{Vortex de Hill thermo-capillaire}

Dans l'étude de Bauer (1982), l'on remarque que la solution obtenue dans le cas d'un gradient thermique axial constant était un tourbillon de Hill de même axe. ${ }^{3}$

On peut obtenir directement le résultat comme suit.

Considérons une goutte sphérique de liquide dont la surface est une séparation de phase de tension capillaire $\sigma$. On suppose que cette tension capillaire est une fonction linéaire de la température :

$$
\sigma=\sigma_{0}+\sigma_{T}\left(T-T_{0}\right)
$$

Le mouvement du liquide est organisé en tourbillon de Hill, mais si l'on suppose que le fluide extérieur est parfait et incompressible, il est au repos.

On peut se poser la question suivante :

Quel champ de température est capable de générer un tourbillon de Hill par suite du mouvement de la surface de la goutte par effet thermo-capillaire?

Pour répondre à cette question on établit les conditions d'équilibre à vérifier entre les forces capillaires et les forces visqueuses à la surface de la sphère.

Au point $M$, le vecteur vitesse est (Figures 2 et 3 , avec $U_{\infty}=V_{0}$ dans le cas de la section 2) :

$$
\overrightarrow{\mathbf{v}}_{i}=\frac{3 V_{0}}{2} \frac{y}{R^{2}}(y \overrightarrow{\mathbf{i}}-z \overrightarrow{\mathbf{k}})=\frac{3 V_{0}}{2}\left[\frac{r^{2}}{R^{2}} \overrightarrow{\mathbf{t}} \sin \theta+\left(\frac{r^{2}}{R^{2}}-1\right) \overrightarrow{\mathbf{n}} \cos \theta\right]=\frac{3 V_{0}}{2}\left(v_{t} \overrightarrow{\mathbf{t}}+v_{n} \overrightarrow{\mathbf{n}}\right)
$$

\footnotetext{
3 Contrairement à la section 2, la goutte sphérique n'est pas en présence d'un écoulement extérieur bien précisé. Cela n'a pas une grande importance si l'on néglige les interaction entre la goutte et les mouvements éventuels en son extérieur.
} 
En $P$, c'est-à-dire pour $r=R$, on a : $\overrightarrow{\mathbf{v}}=\overrightarrow{\mathbf{U}}=-2 \alpha_{i} R^{2} \sin \theta \overrightarrow{\mathbf{e}}_{\theta}=\frac{3 V_{0}}{2} \overrightarrow{\mathbf{t}} \sin \theta$.

L'équilibre des forces en un point de la surface capillaire de la goutte sphérique implique le calcul du taux de déformation tangentiel : $\varepsilon_{t n}=\frac{1}{2}\left(r \frac{\partial\left(U_{t} / r\right)}{\partial r}+\frac{1}{r} \frac{\partial U_{n}}{\partial \theta}\right)$, qui permet ${ }^{4} \mathrm{~d}^{\prime}$ exprimer la contrainte tangentielle $\tau_{t n}=2 \mu \varepsilon_{t n}$.

On a $: \frac{U_{\theta}}{r}=\frac{2 \alpha_{i}}{r}\left(R^{2}-2 r^{2}\right) \sin \theta, \quad U_{r}=2 \alpha_{i}\left(r^{2}-R^{2}\right) \cos \theta$, donc $: \tau_{\theta r}=\tau_{r \theta}=6 \mu R \alpha_{i} \sin \theta$.

Cette contrainte tangentielle est égale à : $\frac{\partial \sigma}{\partial s}=\frac{1}{R} \frac{\partial \sigma}{\partial \theta}=\frac{\sigma_{T}}{R} \frac{\partial T}{\partial \theta}$ (Figure 5).

Il en résulte que $\frac{\partial T}{\partial \theta}=\frac{4 \mu R^{2} \alpha_{i}}{\sigma_{T}} \sin \theta$, soit : $T=T_{0}-\frac{4 \mu R^{2} \alpha_{i}}{\sigma_{T}} \cos \theta$, ou encore : $T=T_{0}-\frac{4 \mu R \alpha_{i}}{\sigma_{T}} z$. On trouve un champ de température à gradient constant.

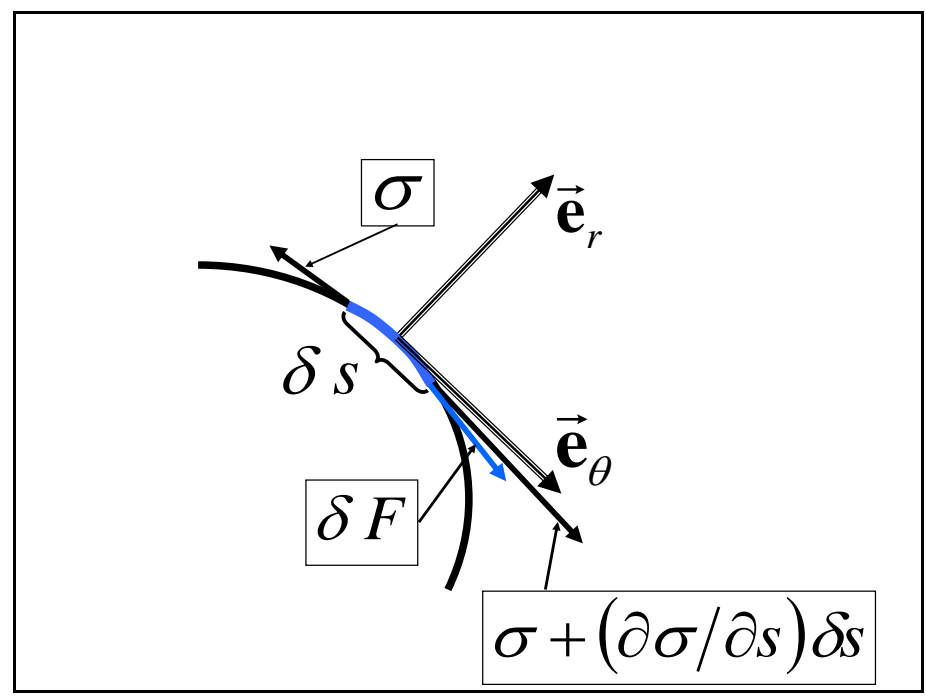

Figure 5. Forces à la surface du liquide. $\delta s=R \delta \theta, \quad F$ : force de frottement visqueux.

On peut donc donner le résultat suivant :

Une goutte liquide sphérique de densité constante, soumise à un gradient de température $\overrightarrow{\mathbf{G}}=\vec{\nabla} T$ uniforme et constant dans une atmosphère au repos, est animée du mouvement intérieur correspondant au vortex de Hill dont la vitesse d'intensité maximale est orientée dans le sens opposé à $\overrightarrow{\mathbf{G}}$ (Figure 6) : $U_{S G}=G \sigma_{T} R / 2 \mu, G$ étant le gradient thermique $G=d T / d z$.

$$
U_{\max }=3 V_{0} / 2=-G \sigma_{T} R / 2 \mu
$$

\footnotetext{
${ }^{4}$ On calcule les efforts de surface à partir du champ de vitesse du tourbillon de Hill en introduisant une viscosité, alors que ce tourbillon est un écoulement de fluide parfait. Cela n'est pas contradictoire si l'on admet qu'il s'agit d'une influence locale et que le corps de l'écoulement est très peu modifié.
} 


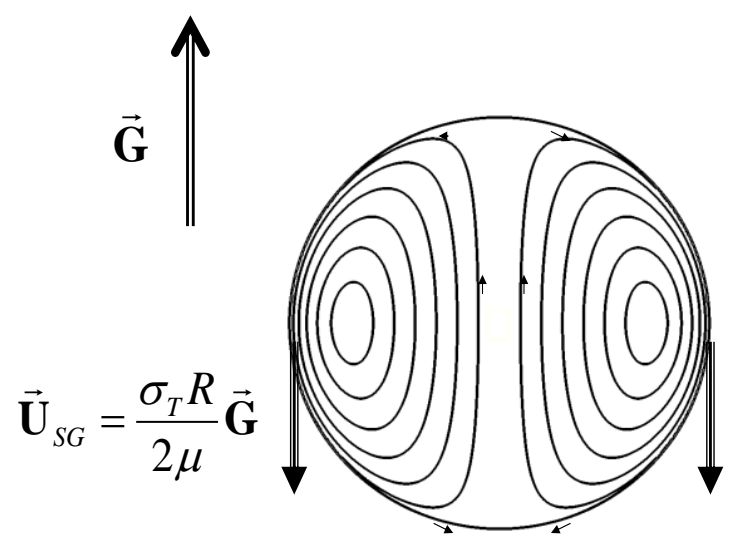

Figure 6. Vortex de Hill généré par un gradient thermique.

\subsection{Autres écoulements thermo-capillaires}

Comme indiqué au début de la section 3.1, Bauer traite aussi d'instabilités dans d'autres cas que celui du gradient thermique axial constant.

Dans son article de 1985 (réf. vi), Bauer étudie les effets combinés de la convection de Marangoni induite par un gradient de température imposé à la surface libre d'une sphère liquide et de la convection naturelle provenant du champ de microgravité résiduelle existant dans un laboratoire spatial en orbite viii. Le cas d'un champ de gravité résiduelle axial constant et linéairement dépendant a été considéré, pour lequel l'équation de Stokes dans l'approximation de Boussinesq a été résolue.

Un nombre de Bond dynamique dérivé du rapport du nombre de Grashof et du nombre de Reynolds basé sur l'écoulement de Marangoni est introduit. Il permet de déterminer la prédominance de l'effet Marangoni si $B o \rightarrow 0$ ou de la convection naturelle si $B o \rightarrow \infty$.

Les effet combiné de Marangoni et de la convection naturelle sont alors étudiés.

Des lignes de courant, des distributions de vitesse radiale et angulaire ont été obtenues analytiquement. D'autre part, les isothermes sont présentées pour différentes distributions de température imposées à la surface libre de la sphère liquide.

Le nombre de Bond dynamique introduit est par définition :

$$
\widetilde{B} o=\rho g \beta R^{2} /\left|\sigma_{T}\right|
$$

La principale influence gravitationnelle est due à l'accélération résiduelle normale au trajet orbital, dans le plan de l'orbite. Elle est créée par l'accélération centrifuge de la station spatiale en orbite circulaire et à l'accélération de Newton.

Lorsque $g$ est constant, on écrit $g=g_{0}$, pour $g$ variable, on a $g=\Omega_{0}^{2} R$ où $\Omega_{0}$ est la vitesse angulaire du centre de masse autour du centre de la terre.

Les équations [12] à [14] s'appliquent toujours, mais l'équation [15] est remplacée par 


$$
\bar{\Delta}\left[\bar{\Delta} \psi-\frac{1}{v} \frac{\partial \bar{\psi}}{\partial t}\right]=-\frac{3 \Omega_{0}^{2} \beta}{v}\left[r^{2} \frac{\partial T}{\partial r} \sin \theta \cos \theta+r \frac{\partial T}{\partial \theta} \cos ^{2} \theta\right] \sin \theta
$$

où $\beta$ est le coefficient de dilatation thermique du liquide.

On utilise alors les formules de récurrence des fonctions de Legendre. Les calculs conduisent à des expressions de la fonction de courant $\psi(r, \theta)$ et des composantes $u(r, \theta), v(r, \theta)$ du vecteur vitesse selon des développements en série de $P_{n}^{0}(\cos \theta)$ et $P_{n}^{1}(\cos \theta)$.

Les constantes intervenant dans ces expressions sont à déterminer selon les conditions limites à la surface de la sphère liquide.

En l'absence d'effets capillaires on obtient la convection naturelle due à la gravité résiduelle présente à l'endroit de la station spatiale où se trouve la goutte. Il faut alors distinguer le cas où liquide est dans un contenant rigide du cas de la surface libre à $\widetilde{B}_{0} \rightarrow \infty$ où l'on peut négliger les effets thermo-capillaires.

On résout aussi les situations où les mouvements internes à la goutte d'origine thermo-capillaire sont importants, et on peut ainsi prévoir les effets de la gravité résiduelle sur ces mouvements.

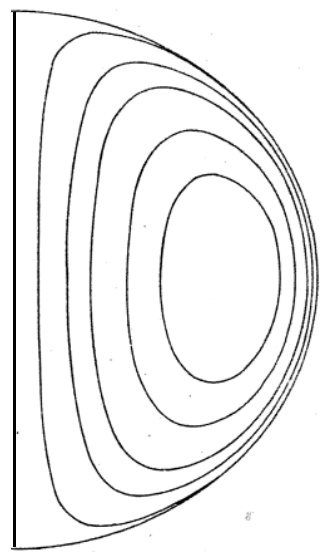

a

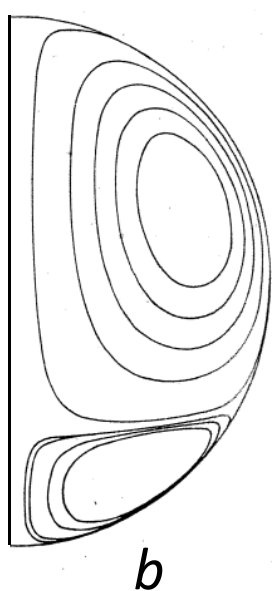

$b$

Figure 7. Lignes de courant d'anneaux tourbillonnaires en présence d'un champ de gravité variable (Bauer 1985): a) pour un champ de température linéaire et b) quadratique.

En ce qui concerne le champ de gravité deux situations sont examinées numériquement : la micro-gravité constante et la micro-gravité à variation linéaire. Quant au champ de température le champ est axisymétrique linéaire $T=T_{0}+T_{1} \cos \theta$ ou mixte linéaire-quadratique $T=T_{0}+T_{1} \cos \theta+T_{2} \cos ^{2} \theta$.

Des résultats des calculs effectués montrent sur la figure 7, la possibilité de différences notables avec les tourbillons de Hill. Des configuration à deux anneaux de surfaces de courant sont observées (figures tirées de l'article de Bauer 1985) avec un champ de gravité résiduelle variable et des champs de température quadratiques pour $T_{2} / T_{1}=2$ avec $\widetilde{B} o=0,10,100$.

Une autre figure de l'article cité montre aussi un cas à deux surfaces toriques avec un champ de gravité résiduelle constant, le même champ de température quadratique et $\widetilde{B} o=10$. 


\section{Conclusion}

Nous avons présenté le tourbillon de Hill comme une structure pouvant apparaître à l'intérieur de gouttes liquides dans deux circonstances : écoulement fluide extérieur uniforme à l'infini, gradient thermique le long d'une direction axiale.

Les hypothèses physiques étaient :

- La sphéricité de la goutte de rayon constant

- Le liquide : incompressible, dilatable ou non, visqueux mais animé d'un mouvement rotationnel de fluide parfait en régime stationnaire

- Le fluide externe : au repos ou animé d'un mouvement uniforme à l'infini, parfait irrotationnel ou localement visqueux

- Concernant l'interface liquide fluide extérieur :

○ Simple surface de contact ou surface de glissement

○ Identité des vitesses : fluides-surface ou seulement liquide-surface

- Avec ou sans tension superficielle fonction de la température

Nous avons eu le soucis de situer le cas particulier du tourbillon de Hill comme cas particulier de l'analyse par séries de fonction de Legendre.

\section{Perspectives :}

Sur le vortex de Hill, il faudra conclure sur la naissance et la dissipation de ce vortex en fournissant des temps caractéristiques ${ }^{\mathrm{ix}, \mathrm{x}}$.

Sur l'instabilité de Marangoni dans une goutte soumise à un champ thermique radial à symétrie sphérique, nous aurons à affiner notre formulation du problème en coordonnées sphériques en nous servant des articles de Hoefsloot et al. xi, xii

Il serait intéressant d'étudier l'effet du vortex thermo-capillaire sur l'évaporation de la goutte ${ }^{\text {xiii, }}$ ${ }^{x i v}$, en particulier en présence de rayonnement thermique ${ }^{\mathrm{xv}}$ ou d'excitation acoustique comme nous l'avons fait avec d'autres phénomènes ${ }^{\mathrm{xvi}}$.

Des chercheurs s'intéressent d'ores et déjà à poursuivre des investigations sur le sujet 5 .

\section{Annexes}

A1. Rappels sur les écoulements irrotationnels 3D de fluides parfaits incompressibles : source, puits, doublet, écoulements de révolution

\section{A1.1. Généralités ${ }^{\text {xvii }}$}

Considérons des écoulements dont le vecteur vitesse dérive d'un potentiel $\phi$. Soit $\overrightarrow{\mathbf{v}}=\vec{\nabla} \phi$ le vecteur vitesse.

L'équation de continuité se résume à $\vec{\nabla} \cdot \overrightarrow{\mathbf{v}}=0$, d'où l'on déduit : $\Delta \phi=0$. La fonction $\phi$ est donc une fonction harmonique.

D'après le deuxième théorème de Bernoulli, portant sur les écoulements irrotationnels (mais pouvant être instationnaires), on a, $\mathcal{V}$ désignant le potentiel dont sont supposées dériver les forces extérieures à distance :

\footnotetext{
5 Notamment à l'Université de Lomé (Togo), et à l'Université of Abomey-Calavi (Bénin).
} 


$$
\mathcal{H}=\frac{\overrightarrow{\mathbf{v}}^{2}}{2}+\int \frac{d p}{\rho}+\mathcal{V}, \text { avec }: \frac{\partial \phi}{\partial t}+\mathcal{H}=C(t)
$$

Nous donnons ci-après trois exemples élémentaires de tels écoulements : l'écoulement uniforme, la source et le doublet.

L'on peut superposer des écoulements puisque les équations sont linéaires.

\section{A1.1.1. Ecoulement uniforme}

L'écoulement uniforme de vitesse $U$ suivant l'axe Ox a pour potentiel de vitesse :

$$
\phi=U x_{3}
$$

\section{A1.1.2. Source et puits}

Une source ponctuelle tridimensionnelle centrée en $\mathrm{O}$ est définie par le potentiel :

$$
\phi=-\frac{D}{4 \pi r}, \quad r^{2}=x_{1}^{2}+x_{2}^{2}+x_{3}^{2}
$$

Le vecteur vitesse est : $\overrightarrow{\mathbf{v}}=\vec{\nabla} \phi=\frac{D}{4 \pi} \frac{\overrightarrow{\mathbf{O M}}}{r^{3}}$.

Pour une source, le débit $D$ est positif : $D>0$, alors que pour un puits, il est négatif : $D<0$.

\section{A1.1.3. Doublet}

Soient une source et un puits symétriques par rapport à l'origine (figure A.1) :

$$
\phi=\frac{D}{4 \pi}\left(\frac{1}{r_{A^{\prime}}}-\frac{1}{r_{A}}\right), \quad \frac{1}{r_{A}}=\left[x_{1}^{2}+x_{2}^{2}+\left(x_{3}-a\right)^{2}\right]^{-\frac{1}{2}} \approx \frac{1}{r}\left[1+\frac{a x_{3}}{r^{2}}+\ldots\right]
$$

On pose : $K=2 D a$, on obtient, lorsque $a \rightarrow 0$ et $D \rightarrow \infty:$

$$
\phi=-\frac{K}{4 \pi} \frac{x_{3}}{r^{3}}=-\frac{K}{4 \pi} \frac{\overrightarrow{\mathbf{e}}_{3} \cdot \overrightarrow{\mathbf{O M}}}{r^{3}}
$$

Il s'agit d'un doublet d'axe $\overrightarrow{\mathbf{e}}_{3}$ et d'intensité $K$.

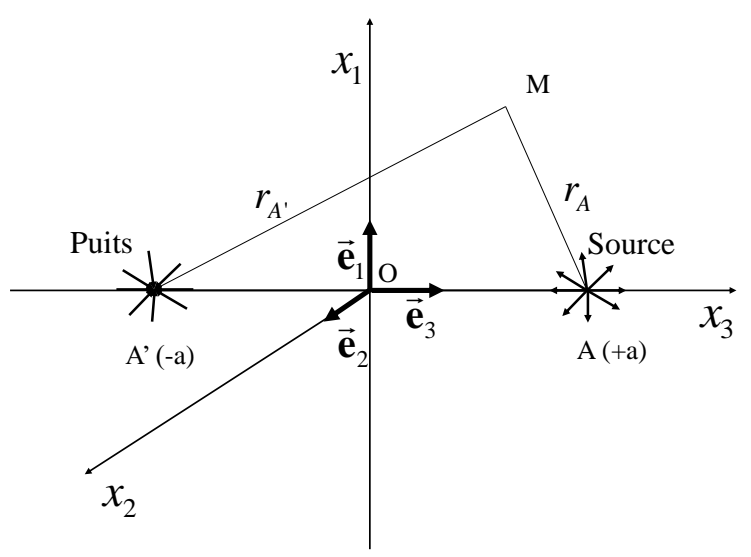

Figure A.1. Doublet dans l'espace 3D: on fait tendre vers zéro la distance 2a entre le puits et la source pour obtenir un doublet localisé à l'origine des axes. 


\section{A1.2. Ecoulements de révolution stationnaires irrotationnels}

On choisit, pour étudier les écoulements de révolution, un système de coordonnées sphériques $r, \theta, \varphi$ disposé comme sur la figure A1.2 par rapport aux coordonnées cartésiennes $O x_{1} x_{2} x_{3}$. Par raison de symétrie l'écoulement est le même dans tout plan yOz passant par l'axe $O z=O x_{3}$, et l'on appelle le vecteur unitaire $\overrightarrow{\mathbf{i}}$ normal à ce plan dans le sens direct.

Il existe alors $\psi(y, z)$ tel que $\overrightarrow{\mathbf{v}}=-\frac{\overrightarrow{\mathbf{i}}}{y} \times \vec{\nabla} \psi$. De plus, l'écoulement étudié étant

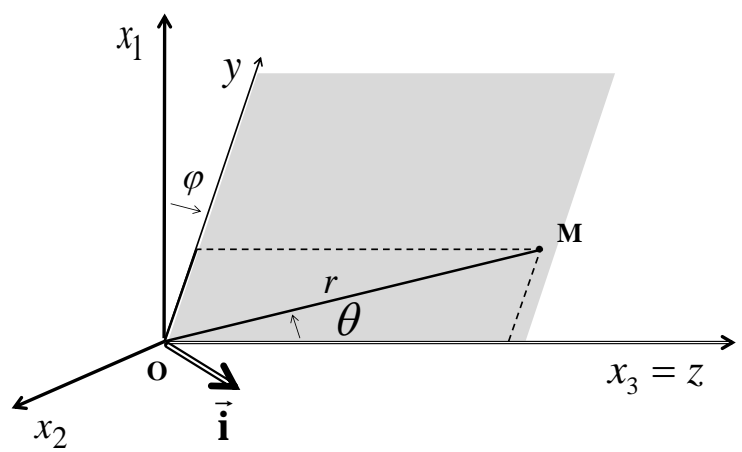

Figure A.2. Le système d'axes de coordonnées dans lequel on étudie l'écoulement de révolution. Les coordonnées sphériques sont $r, \theta, \varphi$ et on considère un plan quelconque y $O z$ passant par l'axe $O z=O x_{3}$, défini par une valeur de l'angle $\varphi$

irrotationnel, on peut écrire : $\overrightarrow{\mathbf{v}}=\vec{\nabla} \phi$. Il s'en suit que :

$$
\frac{\partial \phi}{\partial z}=\frac{1}{y} \frac{\partial \psi}{\partial y}, \frac{\partial \phi}{\partial y}=-\frac{1}{y} \frac{\partial \psi}{\partial z}
$$

$\phi$ vérifie : $\frac{\partial^{2} \phi}{\partial z^{2}}+\frac{1}{y} \frac{\partial}{\partial y}\left(y \frac{\partial \phi}{\partial y}\right)=0$ et $\psi$ vérifie : $\frac{\partial^{2} \psi}{\partial z^{2}}+\frac{1}{y} \frac{\partial}{\partial y}\left(y \frac{\partial \psi}{\partial y}\right)=0$.

\section{A1.3. Exemples}

A1.3.1. Fonctions de courant d'exemples élémentaires

Les potentiels et fonctions de courant de l'écoulement uniforme de vitesse $U_{\infty}$ vérifient :

$$
\phi=U_{\infty} z \Rightarrow \psi=\frac{U_{\infty} y^{2}}{2}+C t e, \operatorname{car} v_{z}=\psi_{, y} / y=U_{\infty} \text { et } v_{y}=\psi_{, z} / y=0 .
$$

Un écoulement de source est défini comme suit par l'expression de son potentiel et de sa fonction de courant :

$\phi=-\frac{D}{4 \pi} \frac{1}{\left(y^{2}+z^{2}\right)^{1 / 2}} \Rightarrow \psi=-\frac{D}{4 \pi} \frac{z}{\left(y^{2}+z^{2}\right)^{1 / 2}}$, soit encore $: \phi=-\frac{D}{4 \pi r}$ et $\psi=-\frac{D}{4 \pi} \cos \theta$

Pour un doublet d'intensité $K$ on a : 
$\phi=-\frac{K}{4 \pi} \frac{z}{\left(y^{2}+z^{2}\right)^{3 / 2}}=-\frac{K}{4 \pi} \frac{\cos \theta}{r^{2}}, \quad \psi=\frac{K}{4 \pi} \frac{y^{2}}{\left(y^{2}+z^{2}\right)^{3 / 2}}=\frac{K}{4 \pi} \frac{\sin ^{2} \theta}{r}$

\section{A1.3.2. Ecoulement autour d'une sphère}

Le système vérifié par $\phi$ et $\psi$ est linéaire, d'où il découle que les combinaisons linéaires de solutions sont possibles.

Par exemple, la superposition d'un écoulement uniforme et d'un doublet donne l'écoulement autour d'une sphère :

$$
\begin{aligned}
& \phi=U_{\infty} z-\frac{K}{4 \pi} \frac{z}{r^{3}}, \quad \psi=\frac{U_{\infty}}{2} y^{2}+\frac{K}{4 \pi} \frac{y^{2}}{r^{3}}, \text { soit, avec } K=-2 \pi U_{\infty} R^{3}: \\
& \phi=U_{\infty} z\left(1+\frac{R^{3}}{2 r^{3}}\right), \quad \psi=\frac{U_{\infty}}{2} y^{2}\left(1-\frac{R^{3}}{r^{3}}\right)
\end{aligned}
$$

Les champs de vitesse qui en résultent sont les suivants :

$$
\begin{aligned}
& v_{z}=\phi_{, z}=\psi_{, y} / y=U_{\infty}\left[1+\frac{R^{3}}{2 r^{5}}\left(y^{2}-2 z^{2}\right)\right] \\
& v_{y}=\phi_{, y}=-\psi_{, z} / y=-\frac{3}{2} U_{\infty} R^{3} \frac{y z}{r^{5}}
\end{aligned}
$$

A la surface de la sphère en $r=R$, on peut écrire :

$U_{x}=\frac{3}{2} U_{\infty} y^{2} / R^{2}, U_{y}=-\frac{3}{2} U_{\infty} x y / R^{2} \Rightarrow \frac{U_{y}}{U_{x}}=-\frac{x}{y}$. Le vecteur vitesse $\overrightarrow{\mathbf{v}}$ est bien tangent à la sphère.

D'autre $\operatorname{part} v=|\overrightarrow{\mathbf{v}}|=\frac{3}{2} U_{\infty}|\sin \theta|$.

Le théorème de Bernoulli donne :

$$
\frac{p}{\rho}+\frac{\mathbf{v}^{2}}{2}=\frac{p_{\infty}}{\rho}+\frac{U_{\infty}^{2}}{2}, \quad p=p_{\infty}+\rho \frac{U_{\infty}{ }^{2}}{2}\left(1-\frac{9}{4} \sin ^{2} \theta\right)
$$

La résultante des efforts s'exerçant sur la sphère s'obtient par intégration de $-p \overrightarrow{\mathbf{n}}$, où $\overrightarrow{\mathbf{n}}$ est la normale extérieure à la surface de la sphère. Par raison de symétrie, cette résultante est donc nulle. La résultante des efforts exercés par un écoulement fluide sur une sphère immobile serait donc nulle.

C'est le paradoxe de d'Alembert. En réalité la résultante n'est pas nulle, car des efforts de frottement s'exercent sur la surface de la sphère du fait de la viscosité, non prise en compte dans le calcul précédent.

\section{A2. Ecoulements rotationnels de fluides parfaits incompressibles}

On ne fait plus ici l'hypothèse d'irrationalité de l'écoulement.

\section{A2.1. Equation de transport du tourbillon}

Si les forces massiques dérivent d'un potentiel, alors le vecteur taux de rotation (ou "tourbillon") $\overrightarrow{\mathbf{\omega}}=\frac{1}{2} \overrightarrow{\mathbf{r o t}} \overrightarrow{\mathbf{v}}$, non nul, est transporté par le mouvement. On a en effet : 


$$
\frac{d \overrightarrow{\mathbf{\omega}}}{d t}=\vec{\nabla} \otimes \overrightarrow{\mathbf{v}} \cdot \overrightarrow{\mathbf{\omega}}
$$

On retrouve ainsi une propriété de la théorie des déformations, suivant laquelle, pour tout vecteur matériel infinitésimal : $\frac{d \delta \overrightarrow{\mathbf{M}}}{d t}=\vec{\nabla} \otimes \overrightarrow{\mathbf{v}} \cdot \delta \overrightarrow{\mathbf{M}}$. Cette propriété s'applique ici au tourbillon.

Cela se démontre comme suit :

L'équation de la quantité de mouvement d'un fluide parfait s'écrit : $\frac{\partial \overrightarrow{\mathbf{v}}}{\partial t}+\overrightarrow{\mathbf{r o t}} \overrightarrow{\mathbf{v}} \times \overrightarrow{\mathbf{v}}+\vec{\nabla} \mathcal{H}=\overrightarrow{\mathbf{0}}$, avec : $\mathcal{H}=\frac{\overrightarrow{\mathbf{v}}^{2}}{2}+\frac{p}{\rho}+V$ en incompressible. En prenant le rotationnel on obtient :

$\frac{\partial \overrightarrow{\mathbf{\omega}}}{\partial t}+\overrightarrow{\mathbf{r o t}}(\overrightarrow{\mathbf{\omega}} \times \overrightarrow{\mathbf{v}})=\overrightarrow{\mathbf{0}}$, ce qui équivaut à l'équation [A.7].

Si à $t$ donné on connaît $\overrightarrow{\boldsymbol{\omega}}$, on en déduit $\overrightarrow{\mathbf{v}}$ et l'équation régissant l'évolution de $\overrightarrow{\boldsymbol{\omega}}$ permettra, si l'on sait la résoudre, de déterminer le champ des vitesses.

Equations permettant de trouver le vecteur vitesse $\overrightarrow{\mathbf{v}}$ :

$\vec{\nabla} \cdot \overrightarrow{\mathbf{v}}=0, \overrightarrow{\operatorname{rot}} \overrightarrow{\mathbf{v}}=2 \overrightarrow{\boldsymbol{\omega}}$ (supposé connu). Si $\overrightarrow{\boldsymbol{\omega}}$ est non nul dans un domaine borné (et nul ailleurs), on peut former une solution particulière (écoulement canonique) engendré par $\overrightarrow{\boldsymbol{\omega}}$ :

$$
\overrightarrow{\mathbf{v}}^{(2)}(M)=-\frac{1}{2 \pi} \int \frac{\overrightarrow{M M^{\prime} \times \overrightarrow{\boldsymbol{\omega}}\left(M^{\prime}\right)}}{|\overrightarrow{M M}|^{3}} d \mathcal{V}\left(M^{\prime}\right)
$$

On peut ajouter à cette solution un écoulement irrotationnel $\overrightarrow{\mathbf{v}}^{(e)}$ (écoulement irrotationnel d'entraînement). C'est possible en particulier si $\overrightarrow{\boldsymbol{\omega}} \cdot \overrightarrow{\mathbf{n}}=0$ aux frontières.

\section{A2.2. Mouvements rotationnels plans}

Soit $\overrightarrow{\boldsymbol{\omega}}=\omega \overrightarrow{\mathbf{k}}, \frac{d \omega}{d t}=0 \quad\left(\right.$ En effet $: \overrightarrow{\mathbf{k}} d \omega / d t=\omega \vec{\nabla} \otimes \overrightarrow{\mathbf{v}} \cdot \overrightarrow{\mathbf{k}}$ mais $\vec{\nabla} \otimes \overrightarrow{\mathbf{v}}$ est dans le plan (x, y) $\rightarrow \frac{d \omega}{d t}=0$. Voir la figure A.2).

Fonction de courant $\psi:$ on a $\overrightarrow{\mathbf{v}}=-\overrightarrow{\mathbf{k}} \times \vec{\nabla} \psi=\overrightarrow{\operatorname{rot}}(\psi \overrightarrow{\mathbf{k}})$.

Remarque : Dans le cas général, $\vec{\nabla} \cdot \overrightarrow{\mathbf{v}}=0 \rightarrow \overrightarrow{\mathbf{v}}=\overrightarrow{\mathbf{r o t}}(\overrightarrow{\mathbf{W}})$ et l'on peut choisir $\overrightarrow{\mathbf{W}}$ tel que $\vec{\nabla} \cdot \overrightarrow{\mathbf{W}}=0$, alors : $\vec{\nabla} \times(\vec{\nabla} \times \overrightarrow{\mathbf{W}})=\vec{\nabla}(\vec{\nabla} \cdot \overrightarrow{\mathbf{W}})-\Delta \overrightarrow{\mathbf{W}} \rightarrow \Delta \overrightarrow{\mathbf{W}}=-2 \overrightarrow{\mathbf{\omega}} . \psi \overrightarrow{\mathbf{k}}$ est un $\overrightarrow{\mathbf{W}}$ particulier, donc $: 2 \overrightarrow{\boldsymbol{\omega}}=-\vec{\nabla} \psi$.

Exemple : Le tourbillon de Rankine. Ce tourbillon résulte de la superposition du mouvement d'un disque solide indéformable de rayon $a$ avec la vitesse angulaire de rotation $\Omega$ et du mouvement d'un tourbillon irrotationnel d'intensité $\Gamma$ extérieur à ce disque. Avec une troisième direction normale au plan cet exemple est représentatif du tourbillon de vidange.

La condition de raccordement en $r=a$ est alors $\Omega=\Gamma / 2 \pi$. Le champ de la fonction de courant est le suivant : 
$\left\{\begin{array}{l}\psi=-(\Gamma / 4 \pi)\left(r^{2} / a^{2}-1\right), r \leq a \\ \psi=-(\Gamma / 2 \pi) \ln r / a, r \geq a\end{array}\right.$

\section{A3. Détail des calculs valables à l'intérieur de la sphère}

Cette annexe se rapporte à la section 2.1.5. La solution dans la zone rotationnelle $(r \leq R)$ s'écrit : $\psi=\alpha r^{2}\left(R^{2}-y^{2}-z^{2}\right)$ et $\omega=5 \alpha r$, avec $\alpha, R$ constants, $\alpha=-3 U_{\infty} / 4 R^{2}$. On a $\psi / \alpha \geq 0$. En posant $r^{2}=y^{2}+z^{2}$ et $z / y=\operatorname{tg} \varphi$, il vient $: \cos ^{2} \varphi=\frac{\psi}{\alpha} \frac{1}{r^{2}\left(R^{2}-r^{2}\right)}$. Les lignes de courant sont représentées sur la figure 4 en coordonnées réduites. Pour $\varphi=0, \cos ^{2} \varphi=1, \alpha r^{2}\left(R^{2}-r^{2}\right)=\psi$, ce qui donne deux valeurs de $r^{2}$, (donc de $r$ ), à condition que $\frac{r^{4}}{4}-\frac{\psi}{\alpha} \geq 0: r^{2}=\frac{R^{2}}{2} \pm \sqrt{\frac{R^{4}}{4}-\frac{\psi}{\alpha}} \geq 0$. Soit $r_{m}^{2}=\frac{R^{2}}{2}-\sqrt{\frac{R^{4}}{4}-\frac{\psi}{\alpha}} \geq 0$ et $r_{M}^{2}=\frac{2 R^{2}}{2}+\sqrt{\frac{R^{4}}{4}-\frac{\psi}{\alpha}} \geq 0$

- $\operatorname{Si} \psi=0$, on trouve $r_{M}=R, r_{m}=0$, ce qui correspond au cercle de centre $\mathrm{O}$, donc à la sphère, qui est une surface de courant, et à son centre. Dans les autres cas on a la condition : $0 \leq \psi / \alpha \leq R^{4} / 4$.

- Si $\psi=\alpha R^{4} / 4$, la ligne de courant se réduit au point $\varphi=0, r=R / \sqrt{2}$.

- Maximum de $\cos ^{2} \varphi=\frac{\psi}{\alpha} \frac{1}{r^{2}\left(R^{2}-r^{2}\right)}$ :

$\cos ^{2} \varphi$ est maximum, à valeur de $\psi$ donnée, pour $r=R \sqrt{2} / 2$, ce qui donne le quart de cercle $\bar{X}_{M}$ de la figure 1b. Pour $\psi=0, \varphi_{M}=\pi / 2$. Pour $\psi / \alpha=R^{2} / 4, \varphi_{M}=0$.

- Minimum de la vitesse :

Le module du vecteur vitesse est égal à $|U|=2 \alpha\left[y^{2} z^{2}+\left(R^{2}-2 y^{2}-z^{2}\right)^{2}\right]^{1 / 2}$. Considérons la quantité $\bar{K}=U^{2} / 4 \alpha^{2} R^{4}$, carré de la vitesse réduite, qui est aussi une énergie cinétique réduite. Son expression en fonction de $\bar{y}=y / R, \bar{z}=z / R$ est : $\bar{K}=\bar{y}^{2}-\frac{3 \bar{\psi}}{4}+\frac{\bar{\psi}^{2}}{16} \frac{1}{\bar{y}^{4}}$ avec $\bar{\psi}=4 \psi / \alpha R^{4}$,fonction de courant réduite. Le minimum de $\bar{K}$ (c'est-à-dire de $U$ ) est obtenu pour $\bar{y}^{2}=\bar{\psi}^{2 / 3} / 2$, ce qui donne le lieu géométrique d'équation $\bar{z}_{U}=1-\bar{y}^{2}-\bar{y} / \sqrt{2}$ représenté sur la Figure 1 b. La valeur de $\bar{K}$ minimum, soit :

$\bar{K}_{m}=0,75\left(\bar{\psi}^{2 / 3}-\bar{\psi}\right)$ est représentée sur la figure 5 . 


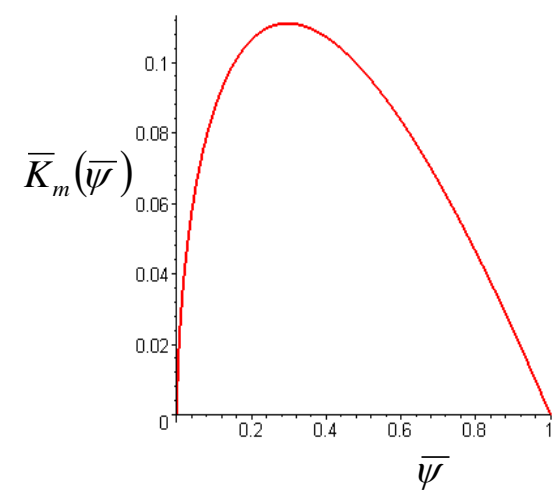

Figure A3. Valeurs du minimum du carré du module de la vitesse $\bar{K}=U_{m}^{2} / 4 \alpha^{2} R^{4}$ avec la fonction de courant réduite $\bar{\psi}=4 \psi / \alpha R^{4}$. On constate que la vitesse ne devient nulle qu'en deux points du quart de plan de la Figure 1b: le point d'arrêt de l'écoulement extérieur $(r=0, z=R)$ et le point : $(r=R / \sqrt{2}, z=0)$ où la surface torique de Hill est réduite à un cercle.

- Circulation du vecteur vitesse :

On sait que si $(\mathrm{C})$ est un contour fermé et $(\mathrm{S})$ une surface régulière par morceaux, ouverte et s'appuyant sur le contour (C), si $\vec{u}$ est une fonction régulière des coordonnées, alors :

$\oint_{C} \vec{u} \cdot \vec{t} d l=\iint_{S} \vec{\nabla} \times \vec{u} \cdot \vec{n} d S=2 \iint_{S} \vec{\omega} \cdot \vec{n} d S$

On peut calculer aisément cette circulation sur la ligne de courant constituée du demi- cercle de rayon $R, y \geq 0$ et du diamètre $y=0,-R \leq z \leq+R$. On obtient en séparant les variables :

$$
\Gamma=20 \alpha \int_{0}^{R} r^{2} d r \int_{\pi / 2}^{0} \cos \varphi d \varphi=-\frac{20}{3} \alpha R^{3}
$$

\section{A4. Polynômes de Legendre et harmoniques sphériques}

\section{A4.1. Rappel sur les polynômes de Legendre}

Les polynômes de Legendre6 ${ }^{6}$ constituent l'exemple le plus simple d'une suite de polynômes orthogonaux ${ }^{7}$. Ce sont des solutions polynomiales $P_{n}(x)$ de l'équation différentielle de Legendre:

$$
\frac{d}{d x}\left[\left(1-x^{2}\right) \frac{d}{d x} P_{n} n(x)\right]+n(n+1) P_{n}(x)=0
$$

\footnotetext{
6 Voir le cours de mathématiques de J. Bass (1968)

7 En mathématiques, une suite de polynômes orthogonaux est une suite infinie de polynômes $p_{0}(x), p_{1}(x), p_{2}(x) \ldots$ à coefficients réels, dans laquelle chaque $p_{n}(x)$ est de degré $n$, et telle que les polynômes de la suite sont orthogonaux deux à deux pour une définition donnée du produit scalaire de fonctions. $\mathrm{Si}\left\langle p_{n}, p_{\mathrm{m}}\right\rangle=0$ pour $n$ différent de $m$, les polynômes sont donc orthogonaux. Le produit scalaire de fonctions le plus simple est l'intégrale du produit de ces fonctions, sur un intervalle borné :
}

$\langle f, g\rangle=\int_{a}^{b} W(x) f(x) g(x) d x$, où $W(x)$ est une fonction poids. 


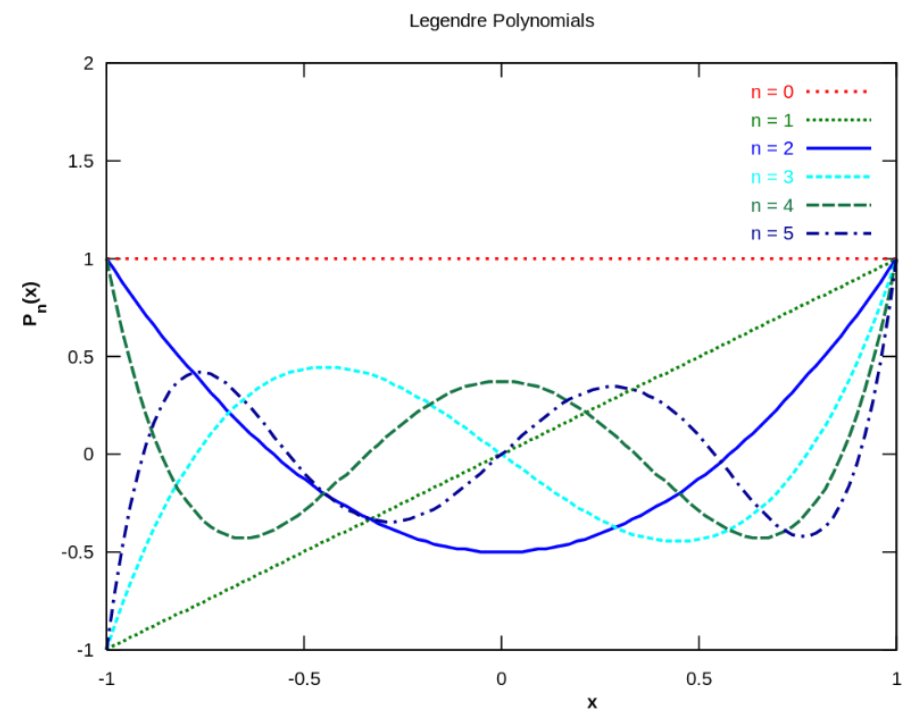

Figure A4. Les polynômes orthogonaux les plus simples sont les polynômes de Legendre pour lesquels l'intervalle d'orthogonalité est ]-1, 1[ et la fonction poids $W(x)$ est la fonction constante de valeur 1. Courbes représentatives des six premiers polynômes de Legendre: $P_{0}(x)=1, P_{1}(x)=x, P_{2}(x)=\left(3 x^{2}-1\right) / 2, P_{3}(x)=\left(5 x^{3}\right.$ -

$$
3 x) / 2, P_{4}(x)=\left(35 x^{4}-30 x^{2}+3\right) / 8, P_{5}(x)=\left(63 x^{5}-70 x^{3}+15 x\right) / 8 \text {. }
$$

dans le cas particulier où le paramètre $n$ est un entier. Les polynômes de Legendre sont définis uniquement pour $x \in[-1 ; 1]$ puisque les points $x= \pm 1$ sont des points singuliers réguliers de cette équation différentielle ${ }^{1}$.

Les courbes représentatives des six premiers polynômes de Legendre: $P_{0}(x)=1, P_{1}(\mathrm{x})=x$, $P_{2}(x)=\left(3 x^{2}-1\right) / 2, P_{3}(x)=\left(5 x^{3}-3 x\right) / 2, P_{4}(x)=\left(35 x^{4}-30 x^{2}+3\right) / 8, P_{5}(x)=\left(63 x^{5}-70 x^{3}+15 x\right) / 8$ apparaissent sur la figure 1 .

Ces polynômes orthogonaux ont de nombreuses applications tant en mathématiques, par exemple pour la décomposition d'une fonction en série de polynômes de Legendre, qu'en physique, où l'équation de Legendre apparaît naturellement lors de la résolution des équations de Laplace ou de Helmholtz en coordonnées sphériques.

\section{A4.2. Les harmoniques sphériques ${ }^{8}$}

Soit l'opérateur $\mathcal{L}^{2}=-\frac{1}{\sin \theta} \frac{\partial}{\partial \theta}\left(\sin \theta \frac{\partial}{\partial \theta}\right)-\frac{1}{\sin ^{2} \theta} \frac{\partial^{2}}{\partial \varphi^{2}}$. Les harmoniques sphériques sont telles que $\mathcal{L}^{2} Y_{l}^{m}(\theta, \varphi)=-l(l+1) Y_{l}^{m}(\theta, \varphi)$.

On montre qu'alors : $Y_{l}^{m}(\theta, \varphi)=P_{l, m}(\cos \theta) e^{i m \varphi}$, où $P_{l, m}(\cos \theta)$ est un polynôme de Legendre.

L'équation aux valeurs propres devient une équation différentielle d'ordre deux pour la fonction $P_{l}^{m}(\cos \theta):-\frac{1}{\sin \theta} \frac{d}{d \theta}\left(\sin \theta \frac{d P_{l, m}(\cos \theta)}{d \theta}\right)+\frac{m^{2}}{\sin ^{2} \theta} P_{l, m}(\cos \theta)=E_{l, m} P_{l, m}(\cos \theta)$.

Le changement de variable $x=\cos \theta$ conduit à l'équation différentielle généralisée de Legendre:

$$
-\frac{d}{d x}\left(\left(1-x^{2}\right) \frac{d P_{l, m}(x)}{d \theta}\right)+\frac{m^{2}}{1-x^{2}} P_{l, m}(x)=E_{l, m} P_{l, m}(x)
$$

\footnotetext{
8 Voir par exemple le site de Wikipédia sur cette question
} 
Les valeurs propres de cette équation sont indépendantes de $m$ :

$$
E_{l, m}=l(l+1)
$$

Les fonctions propres $P_{l, m}(x)$ se construisent à partir des polynômes de Legendre qui sont des fonctions propres de l'équation différentielle ordinaire de Legendre, correspondant au cas $m=0$ :

$$
-\frac{d}{d x}\left[\left(1-x^{2}\right) \frac{d}{d x} P_{l}(x)\right]=l(l+1) P_{l}(x)=0
$$

On a la formule génératrice d'Olinde Rodrigues :

$$
P_{l}(x)=\frac{1}{2^{l} l !} \frac{d^{l}}{d x^{l}}\left(x^{2}-1\right)^{l}
$$

- Pour $l$ non entier, on trouve des solutions tendant vers l'infini quand $x$ tend vers $(-1)$

- Les seules solutions admissibles sont donc celles définies pour $l$ entier

- On trouve : $P_{l}(x)=\frac{(-l)^{m}}{2^{l} l !}\left(1-x^{2}\right)^{m / 2} \frac{d^{l+m}}{d x^{l+m}}\left(x^{2}-1\right)^{l}$

- $P_{l}^{m}$ et $P_{l}^{-m}$ sont deux fonctions propres correspondant à la même valeur propre $l(l+1)$, mais en réalité, ils sont proportionnels : $P_{l,(-m)}(x)=\frac{(n-m) !}{(n+m) !} P_{l, m}(x)$.

- Les harmoniques sphériques formant une base orthogonales sur la sphère unité, toute fonction continue $f(\theta, \varphi)$ se décompose en une série d'harmoniques sphériques :

$f(\theta, \varphi)=\sum_{l=0}^{l=+\infty} \sum_{m=-l}^{m=+l} C_{l}^{m} Y_{l}^{m}(\theta, \varphi)$, où $l$ et $m$ sont des indices entiers, $C_{l}^{m}$ est un coefficient constant qui souvent en mathématiques prend le nom de coefficient de Fourier généralisé relativement à cette base (le développement en harmoniques sphériques est l'équivalent, appliqué aux fonctions angulaires, du développement en séries de Fourier pour les fonctions périodiques).

- $Y_{l}^{m}$ est la partie réelle d'une fonction complexe $\underline{Y_{l}^{m}}: Y_{l}^{m}(\theta, \varphi)=\mathfrak{R}\left(\underline{Y_{l}^{m}(\theta, \varphi)}\right)$.

$\underline{Y_{l}^{m}}$ est appelée "fonction associée de Legendre" et est définie par

$\underline{Y_{l}^{m}}(\theta, \varphi)=\sqrt{\frac{2(l-m) !}{(l+m) !}} P_{l, m}(\cos \theta) e^{i m \varphi}$, où $P_{l, m}(\cos \theta)$ est le polynôme de Legendre :

$P_{l, m}(X)=\frac{(-1)^{m}}{2^{l} l !}\left(1-X^{2}\right)^{m / 2} \frac{\partial^{m+1}}{\partial X^{m+1}}\left[\left(X^{2}-1\right)^{l}\right]$.

On a par exemple les fonctions de la figure 1.

Les fonctions $Y_{l}^{m}(\theta, \varphi)$ présentent de plus en plus de symétries au fur et à mesure que $l$ croît (sauf lorsque $l=0$, puisque $Y_{0}^{0}$ est une fonction constante et décrit donc une sphère).

- Harmoniques sphériques généralisées 
Les harmoniques sphériques généralisées sont définies sur la sphère $\mathrm{S}_{3}$. La normalisation des harmoniques sphériques conduit à l'expression finale :

$$
Y_{l}^{m}(\theta, \varphi)=\sqrt{\frac{(2 l+1)(l-m) !}{4 \pi(l+m) !}} P_{l,|m|} \cos \theta e^{i m \varphi}
$$

\section{Références}

${ }^{i}$ Abramzon, B. \& Sirignano, W. A. : Droplet vaporization model for spray combustion calculations, Int. J. Heat Mass Transfer, 32, No 9, 1605-1618, 1989. DOI: 10.1016/0017-9310(89)90043-4.

${ }^{i i}$ Lamb, H.: Hydrodynamics, Cambridge University Press, Cambridge, 1945.

iii Germain, P. : Mécanique, t. 1, Ellipses, Ecole Polytechnique, Palaiseau, 1986.

iv Prud'homme, R., Ecoulements et réactions chimiques 1- collection Mécanique des fluides, Hermès-Lavoisier, Cachan, 2012. http://www.lavoisier.fr/livre/notice.asp?ouvrage=2649728\&pos=1

${ }^{v}$ Bauer, H.F. : Marangoni convection in a freely floating liquid sphere due to axial temperature field. Ing. Arch., 52 , 263-273, 1982.

${ }^{\mathrm{vi}}$ Bauer, H.F. : Combined residual natural and Marangoni convection in a liquid sphere subjected to a constant and variable micro-gravity field. Zamm Z. angew. Math. Mech. 65 10, 461-470, 1985.

${ }^{v i i}$ Bauer, H.F.\& Eidel, W. : Marangoni convection in a spherical liquid system. Acta Astronautica, 15, 275-290, 1987.

viii Lefebvre D., Microgravité dans l'ISS, TangenteX.com septembre 2018 http://www.tangentex.com/Microgravite.htm

${ }^{\text {ix }}$ Gharib M., Rambod E., Shariff K.: A universal time scale for vortex ring formation. J. Fluid Mech., 360:121-140, 1998.

${ }^{\mathrm{x}}$ Chung, J.N.: The motion of particles inside a droplet. Transactions of the ASME, 104, 438-445, August 1982.

${ }^{x i}$ Hoefsloot, H.C.J., Hoostraten, H.W., Janssen, L.P.B.M.: Marangoni instability in a confined between two concentric spherical surfaces liquid layer under zero-gravity conditions. Applied Scientific research, 47, 357-377 (1990).

${ }^{\text {xii }}$ Hoefsloot, H.C.J., Hoostraten, H.W., Janssen, L.P.B.M., Knobbe, J.W.: Grows factors for Marangoni instability in a spherical liquid layer under zero-gravity conditions. Applied Scientific research, 49, 161-173, Kluwer Academic Publishers (1992).

xiii Shih, A.T., Megaridis, C.M.: Suspended droplet evaporation modeling in a laminar convective environment. Combustion and Flame, 102: 256-270, 1995.

xiv Shih, A.T., Megaridis, C.M.: Thermocapillary flow effects on convective droplet evaporation. Int. J. Heat Mass Transfer, 39, N², pp. 247-257, 1996.

${ }^{\mathrm{xv}}$ Niazmand, H., Ambarsooz, M., Effect of slip and Marangoni convection on single fuel droplet heat-up in the presence of thermal radiation. ICHEC 2009, Kish Island, I.R. Iran.

${ }^{\text {xvi }}$ Mauriot, Y., Prud'homme, R., Assessment of evaporation equilibrium and stability concerning an acoustically excited drop in combustion products. C. R. Mecanique Académie des sciences. 342, pp. 240-253, 2014. https://doi.org/10.1016/j.crme.2014.02.004

xvii Germain, P.: Mécanique des milieux continus, Masson, Paris, 1962. 